\title{
Migraine: another headache for psychiatrists?
}

\author{
C. MAJELLA CAHILL and KIERAN C. MURPHY
}

The publication in early 2004 of a revised classification system for headache by the International Headache Society should be considered relevant to the practice of psychiatrists and other mental health specialists (Headache Classification Subcommittee of the International Headache Society, 2004). Migraine is a chronic paroxysmal disorder characterised by stereotypical attacks of headache, focal neurological symptoms or a combination of both. The most commonly diagnosed migraine subtypes are migraine without aura and migraine with aura (to be known henceforth as 'typical aura with migraine headache'), which have a joint population prevalence in Europe and North America of approximately 10-12\%. The updated diagnostic criteria for these and other headache disorders have been published in Cephalalgia (2004), 24 (suppl. 1) (online at http://216.25.100.131/ ihscommon/guidelines/pdfs/ihc_II_main_no_ print.pdf). Migraine imposes a substantial burden, not only on individual headache sufferers, but also on society at large. The World Health Organization recently classified migraine as the 19th leading cause of disability worldwide (World Health Organization, 2001: Fig. 2.3). In addition, primary care pharmaceutical expenditure in the UK is estimated at $£ 69.6$ million per annum (R. Harkins, personal communication, 2004), and migraine is conservatively estimated to cost the UK economy in excess of $£ 750$ million per annum in terms of lost productivity (Cull et al, 1992).

\section{MIGR AINE, AFFECTIVE DISORDER AND ANXIETY DISORDER}

Many people experience changes in mood or behaviour as a prodromal or accompanying feature of their migraine attacks. Complaints of depression, euphoria, irritability, anxiety, hyperactivity, poor concentration and anorexia or increased appetite are common (Silberstein \& Young, 1995).
In addition, several studies have reported significant associations between migraine, affective disorders and anxiety disorders. Two of the most important were longitudinal epidemiological studies of young adult populations using standardised operational criteria for the diagnosis of both migraine and psychiatric disorder. Merikangas et al (1990) found elevated 1-year prevalence rates for a wide range of psychiatric disorders in people with migraine ('migraineurs') compared with 'non-migraineurs', and reported odds ratios of 2.2 (95\% CI 1.14.8) for major depressive disorder, 2.9 (95\% CI 1.1-8.6) for bipolar spectrum disorders, 5.3 (95\% CI 1.8-15.8) for generalised anxiety disorder, 3.3 (95\% CI 0.8 13.8) for panic disorder, $2.4(95 \%$ CI 1.1-5.1) for simple phobia and 2.0 (95\% CI 1.1-10.9) for social phobia. In addition, Breslau et al (1991) found that lifetime prevalence rates of dysthymia, major depressive disorder, bipolar affective disorder, generalised anxiety disorder, panic disorder and phobia were significantly elevated among people with migraine compared with non-migraineur controls. Several studies have also reported a significant correlation between major depression and anxiety disorders in people with migraine. Breslau et al (1991) found that $88 \%$ of those with migraine and major depression reported a history of at least one anxiety disorder. Similarly, Merikangas et al (1990) reported that people with both major depression and an anxiety disorder were at significantly greater risk of migraine than those with depression alone. Retrospective reports suggested that the onset of anxiety disorder generally preceded the development of migraine, whereas major depression occurred after the onset of migraine.

\section{MECHANISMS OF COMORBIDITY}

A number of studies have investigated potential mechanisms of comorbidity between migraine and affective and anxiety disorders, with much of the research focusing on the relationship between migraine and major depressive disorder. Possible explanations for this association include the following:

(a) migraine and major depressive disorder may be causally related, i.e. migraine may cause depression or conversely be caused by depression;

(b) migraine and major depressive disorder may share a common genetic and/or environmental pathophysiology;

(c) migraine and depression may be different phenotypic expressions of the same disorder.

Breslau \& Davis (1992) reported that the excess risk of developing major depressive disorder was unrelated to whether the person with migraine had experienced a recent attack (within 1 year) or not. A further study by Breslau et al (2003) reported a bidirectional relationship between migraine and major depressive disorder, each increasing the risk for the subsequent first onset of the other. These findings are not supportive of a simple causal relationship between migraine and major depressive disorder, but instead support the hypotheses that these disorders share common pathogenetic mechanisms or occur as different phenotypic expressions of the same disorder. The paroxysmal nature of both migraine attacks and affective episodes, their similar pattern of evolution over time, and their partially overlapping treatment responsivity (see below) lend further support to a shared mechanism hypothesis (Post \& Silberstein, 1994).

The specificity of the migraine-major depression association has been questioned, with suggestions that headache severity and frequency, rather than headache subtype, may determine the relationship with psychiatric disorder. However, the bidirectional association between migraine and major depressive disorder observed by Breslau et al (2003) was specific to migraine and was not observed in relation to other types of headache of similar severity. Thus, although migraine is not exclusively associated with depression, the nature of its association with depression appears to differ from that seen in other severe headache types.

Despite evidence supporting a significant genetic basis to both migraine and depression, Merikangas et al (1988, 
1993), using family study data, found no evidence for the co-segregation of these disorders. The relatives of probands with depression alone did not have elevated rates of migraine, and the relatives of probands with migraine exhibited elevated rates of depression only if migraine was also present. To our knowledge, there has been no twin study examining the co-segregation of migraine and major depression. Thus, the limited data currently available are not supportive of a common genetic aetiology.

Dysregulation of serotonergic neurotransmission has been postulated to have a key role in the pathogenesis of both major depression and migraine. Most of the evidence supports an underactivation of serotonergic function in depression, although the cause of this dysfunction has yet to be determined. Altered interactions between serotonergic and other neurobiological systems probably mediate the symptoms of affective illness expressed (Ressler \& Nemeroff, 2000). Changes in the circulating levels of serotonin and its metabolites during migraine attacks, the ability of serotonin-releasing agents to trigger migraine and the efficacy of certain serotonin receptor ligands in the treatment of migraine provide indirect evidence for the involvement of this neurotransmitter in migraine pathophysiology. In addition, persistent brain-stem activity in the region of the locus ceruleus, dorsal raphe nucleus and periaqueductal grey matter has been demonstrated during spontaneously occurring migraine attacks in humans (Weiller et al, 1995), raising the possibility of a 'migraine generator' in this area. To date, few studies have examined the neurobiology underlying the comorbid expression of migraine and depression, and further studies are required to identify these mechanisms.

The nature of the association between migraine and other specific anxiety or bipolar spectrum disorders has been less comprehensively researched. A bidirectional relationship has been reported for panic disorder, although this appears to be non-specific for migraine and applies equally to headache types of similar severity (Breslau et al, 2001).

\section{CLINICAL IMPLICATIONS FOR PSYCHIATRISTS}

Recognition of the significantly elevated levels of psychopathological disorder associated with migraine is important for a number of reasons, which include mental health service use as well as diagnosis, prognosis and treatment.

\section{Use of mental health services}

Migraine is associated with excess levels of health care resource use, a significant proportion of which burden is borne by the psychiatric services. In US epidemiological studies psychiatric consultation rates were significantly higher among people with migraine compared with control populations. For example, Edmeads \& Mackell (2002) reported that $7 \%$ of people with migraine had consulted a psychiatrist in the 6 months prior to interview, compared with $3 \%$ of a non-migraineur sample $(P<0.001)$. In addition, Breslau \& Davis (1993) reported an elevated 1-year rate of mental health specialist use $(24.2 \% v$. $8.9 \%, P<0.001)$ and an increased lifetime rate of psychiatric hospitalisation $(6.3 \% v$. $2.8 \%, P=0.04)$ in those with migraine.

\section{Diagnosis}

Occasionally, psychiatric symptoms occurring as part of the migraine prodrome or attack may dominate the clinical presentation and require differentiation from a primary psychiatric disorder (Silberstein $\&$ Young, 1995). Another diagnostic challenge when comorbid disease occurs is to recognise that more than one disease may simultaneously be present. Thus, the presence of migraine should increase the index of suspicion for associated psychiatric disorder and, conversely, those presenting for psychiatric treatment should be questioned about the possibility of comorbid migraine, since this may have both therapeutic and prognostic implications.

\section{Treatment}

Comorbidity may provide therapeutic opportunities or impose therapeutic limitations. Although no study has examined the efficacy of pharmacological agents for the simultaneous treatment of both migraine and psychiatric disorder, a number of drug classes may be beneficial in both conditions. Evidence-based guidelines advocate the use of amitriptyline and sodium valproate in the prophylaxis of migraine, although other antidepressant, anticonvulsant and antipsychotic agents may also have a part to play in migraine treatment (Ramadan et al, 2000). Relaxation training, stress management training, biofeedback therapy and cognitive-behavioural therapy are also of proven benefit in migraine prophylaxis (Campbell et al, 2000). Where separate pharmacological agents are required for the treatment of migraine and a comorbid psychiatric disorder, consideration must be given to a number of factors: these include the potential for the treatment of one disorder to exacerbate the other, and the potential for drug interactions; for example, concerns have been raised about the potential of the triptan class of antimigraine agents, which are $5-\mathrm{HT}_{1 \mathrm{~B} / \mathrm{D}}$ agonists, to cause a central serotonergic syndrome when used in combination with other serotonergic agents, such as monoamine oxidase inhibitors, selective serotonin reuptake inhibitors and tricyclic antidepressants, or lithium (Lane \& Baldwin, 1997).

\section{Prognosis}

There is evidence to suggest that patients with comorbid migraine and psychiatric disorder may experience a more severe or complex clinical course, with greater impairment of health-related quality of life, than those with only one of these disorders (Lipton et al, 2000; Fasmer \& Oedegaard, 2001; Breslau et al, 2003). Of particular concern is the finding of an elevated risk of suicide in association with migraine, with a reported odds ratio for suicide attempts of 3.0 (95\% CI 1.2-8.0) (Breslau et al, 1991; Breslau, 1992). Unsurprisingly, both migraine with and without aura were associated with an increased odds ratio for suicidal ideation and attempts when associated with major depressive disorder. However, the association between migraine with aura and suicidal ideation and attempts remained significant even after controlling for the presence of psychiatric disorder: odds ratio for suicide attempt 4.3 (95\% CI 1.2-15.7); odds ratio for suicidal ideation 2.4 (95\% CI 1.1-5.3). Furthermore, Breslau (1992) reported that the risk for suicide attempt in major depressive disorder was significantly elevated in the presence of migraine with aura. The reported odds ratio for suicide attempts in major depression alone was $7.8(95 \% \mathrm{CI}$ 3.5-17.1) compared with 23.2 (95\% CI 8.4-63.8) for major depression and migraine with aura.

\section{IMPLICATIONS FOR FUTURE PR ACTICE}

Migraine, a relatively common disorder, is strongly associated with a number of 
affective and anxiety disorders. This has implications both for the individual headache sufferer and for mental health service providers. Thus, psychiatrists and other mental health specialists should familiarise themselves with the revised diagnostic criteria for the various migraine subtypes. In common with people with other neuropsychiatric disorders, patients with migraine benefit most from treatment within the context of a multidisciplinary team that includes neurologists, psychiatrists and psychologists. In our experience, such a model facilitates both improved diagnostic accuracy and a more coordinated approach to treatment. In our experience, feedback from patients suggests that this is also the approach favoured by people with migraine and their relatives.

\section{DECLARATION OF INTEREST}

None.

\section{REFERENCES}

Breslau, N. (1992) Migraine, suicidal ideation, and suicide attempts. Neurology, 42, 392-395.

Breslau, N. \& Davis, G. C. (1992) Migraine, major depression and panic disorder: a prospective epidemiologic study of young adults. Cephalalgia, 12 85-90.

Breslau, N. \& Davis, G. C. (1993) Migraine, physical health and psychiatric disorder: a prospective epidemiologic study in young adults. Journal of Psychiatric Research, 27, 211-221.

Breslau, N., Davis, G. C. \& Andreski, P. (1991)

Migraine, psychiatric disorders and suicide attempts: an epidemiologic study of young adults. Psychiatry Research, 37, II-23.

C. MAJELLA CAHILL, MRCPsych, KIERAN C. MURPHY, MRCPsych, PhD, Department of Psychiatry, Royal College of Surgeons in Ireland, Education and Research Centre, Beaumont Hospital, Dublin, Ireland

Correspondence: Dr C. Majella Cahill, Department of Psychiatry, Beaumont Hospital, Dublin 9, Ireland. Tel: + 353 (0)। 8093354; fax: + 353 (0I)। 8376982; e-mail: majcahill@rcsi.ie

(First received 3 March 2004, accepted II May 2004)

Breslau, N., Schultz, L. R., Stewart, W. F., et al (200I) Headache types and panic disorder: directionality and specificity. Neurology, 56, 340-344.

Breslau, N., Lipton, R. B., Stewart, W. F., et al (2003) Comorbidity of migraine and depression: investigating potential etiology and prognosis. Neurology, 60, $1308-1312$.

Campbell, J. K., Penzien, D. B. \& Wall, E. M. (2000) Evidence-Based Guidelines for Migraine Headache: Behavioral and Physical Treatments. Saint Paul, MN: American Academy of Neurology. Available at http: // www.aan.com/professionals/

Cull, R. E., Wells, N. E. J. \& Miocevich, M. L. (1992) The economic cost of migraine. British journal of Medical Economics, 2, 103-115.

Edmeads, J. \& Mackell, J. A. (2002) The economic impact of migraine: an analysis of direct and indirect costs. Headache, 42, 501-509.

Fasmer, O. B. \& Oedegaard, K. J. (200I) Clinical characteristics of patients with major affective disorders and comorbid migraine. World Journal of Biological Psychiatry, 2, 149-155.

Headache Classification Subcommittee of the International Headache Society (2004) The International Classification of Headache Disorders: 2nd edition. Cephalalgia, 24 (suppl. I), 9-160. http: // 216.25.100.131/ihscommon/guidelines/pdfs/ihc_Il_main _ no_print.pdf

Lane, R. \& Baldwin, D. (1997) Selective serotonin reuptake inhibitor-induced serotonin syndrome: review. Journal of Clinical Psychopharmacology, 17, 208-221.

Lipton, R. B., Hamelsky, S. W., Kolodner, K. B., et al (2000) Migraine, quality of life, and depression: a population-based case-control study. Neurology, 55, 629-635
Merikangas, K. R., Risch, N. J., Merikangas, J. R., et al (1988) Migraine and depression: association and familial transmission. Journal of Psychiatric Research, 22, 119-129.

Merikangas, K. R., Angst, J. \& Isler, H. (1990) Migraine and psychopathology: results of the Zurich Cohort Study of young adults. Archives of General Psychiatry, 47, 849-853.

Merikangas, K. R., Merikangas, J. R. \& Angst, J. (1993) Headache syndromes and psychiatric disorders: association and familial transmission. Journal of Psychiatric Research, 27, 197-210.

Post, R. M. \& Silberstein, S. D. (1994) Shared mechanisms in affective illness, epilepsy, and migraine. Neurology, 44 (suppl. 7), S37-S47.

Ramadan, N. M., Silberstein, S. D., Freitag, F. G., et al (2000) Evidence-based Guidelines for Migraine Headache in the Primary Care Setting: Pharmacological Management for Prevention of Migraine. Saint Paul, MN American Academy of Neurology. Available at http: //www.aan.com/professionals/

Ressler, K. J. \& Nemeroff, C. B. (2000) Role of serotonergic and noradrenergic systems in the pathophysiology of depression and anxiety disorders. Depression and Anxiety, 12 (suppl. I), S2-S19.

Silberstein, S. D. \& Young, W. B. (1995) Migraine aura and prodrome. Seminars in Neurology, 15, 175-182.

Weiller, C., May, A., Limmroth, V., et al (1995) Brain stem activation in spontaneous human migraine attacks. Nature Medicine, I, 658-660.

World Health Organization (200I) The World Health Report 2001: Mental Health: New Understanding, New Hope. Geneva: WHO. http: / wwww. who. int/whr200I/ 2001/main/en/chapter2/002d.htm 